\title{
Vers un compte satellite des institutions sans but lucratif (ISBL) en France (II ${ }^{\mathbf{e}}$ partie) \\ Towards a satellite account of nonprofit institutions (NPIs) in France (part two)
}

\section{Edith Archambault et Philippe Kaminski}

Numéro 294, novembre 2004

URI : https://id.erudit.org/iderudit/1022019ar

DOI : https://doi.org/10.7202/1022019ar

Aller au sommaire du numéro

Éditeur(s)

Institut de l'économie sociale (IES)

ISSN

1626-1682 (imprimé)

2261-2599 (numérique)

Découvrir la revue

Citer cet article

Archambault, E. \& Kaminski, P. (2004). Vers un compte satellite des institutions sans but lucratif (ISBL) en France (II ${ }^{\mathrm{e}}$ partie). Revue internationale de

l'économie sociale, (294), 11-26. https://doi.org/10.7202/1022019ar
Résumé de l'article

Que la comptabilité nationale permette d'évaluer le poids économique des activités d'économie sociale constitue un enjeu pour l'ensemble des organisations du secteur. Même si cet objectif reste encore hors d'atteinte, une avancée significative a été accomplie, puisque l'Union européenne incite les États-membres à mettre en oeuvre, dans le système des comptes nationaux, le " Manuel des institutions sans but lucratif " (ISBL) tel qu'adopté par l'ONU en 2002. Autoriser une comparaison à l'échelle internationale n'est pas le moindre mérite du compte satellite des ISBL qui, pour les auteurs, ouvre la voie au compte satellite de l'économie sociale pour lequel milite et travaille l'Addes depuis plus de vingt ans. Avant de s'intéresser au « Manuel », l'article a dans une première partie précisé les concepts qui prévalent dans le champ de l'économie sociale et les différentes acceptions dans les pays. Actuellement, seules les institutions sans but lucratif au service des ménages (ISBLSM) sont lisibles dans les comptes nationaux. Comment passer au compte des ISBL ? Comment situer l'ISBL par rapport à l'association? Autant de questions qui sont explorées dans cette importante contribution, présentée au colloque de l'Addes le 15 décembre 2003, que nous publions en deux parties (la première partie est parue dans le numéro 293). 


\section{VERS UN COMPTE SATELLITE DES INSTITUTIONS SANS BUT LUCRATIF (ISBL) EN FRANCE}

(IIle partie)

(*) Présidente de l'Association pour le développement de la documentation en économie sociale (Addes). $\left.{ }^{* *}\right)$ Vice-président de l'Addes. par Edith Archambault ${ }^{(*)}$ et Philippe Kaminski ${ }^{(*)}$

Que la comptabilité nationale permette d'évaluer le poids économique des activités d'économie sociale constitue un enjeu pour l'ensemble des organisations du secteur. Même si cet objectif reste encore hors d'atteinte, une avancée significative a été accomplie, puisque l'Union européenne incite les Etats-membres à mettre en cuvre, dans le système des comptes nationaux, le "Manuel des institutions sans but lucratif» (ISBL) tel qu'adopté par l'ONU en 2002. Autoriser une comparaison à l'échelle internationale n'est pas le moindre mérite du compte satellite des ISBL qui, pour les auteurs, ouvre la voie au compte satellite de l'économie sociale pour lequel milite et travaille l'Addes depuis plus de vingt ans. Avant de sintéresser au "Manuel ", l'article a dans une première partie précisé les concepts qui prévalent dans le champ de l'économie sociale et les différentes acceptions dans les pays. Actuellement, seules les institutions sans but lucratif au service des ménages (ISBLSM) sont lisibles dans les comptes nationaux. Comment passer au compte des ISBL? Comment situer l'ISBL par rapport à l'association? Autant de questions qui sont explorées dans cette importante contribution, présentée au colloque de l'Addes le 15 décembre 2003, que nous publions en deux parties (la première partie est parue dans le numéro 293).

\section{Les ISBL et ISBLSM dans le cadre central de la comptabilité nationale et dans le compte satellite des ISBL}

\footnotetext{
Remarques préalables

Cette troisième partie reprend pour l'essentiel des documents rédigés par Sybille Mertens, aux fins de présentation du projet de compte satellite des associations en Belgique. Adaptée au contexte français, elle n'ambitionne que de préciser comment sont définis les concepts intervenant dans notre propre projet de compte satellite des ISBL. Pour faciliter la lecture, les notes d'origine ont été réintégrées dans le corps du texte, dans des paragraphes en italique avec la mention [SYB]. Lorsque la mention est [PHK], c'est qu'il s'agit de notes spécifiquement rédigées pour le document Addes. Nous renouvelons ici tous nos remerciements à Sybille Mertens, tant pour son aide précieuse que pour sa cordialité sans faille.
} 
La comptabilité nationale, dont les concepts sont définis au niveau international par le SCN (1993), puis au niveau européen par le SEC (1995), définit la catégorie des ISBL, mais n'en évalue qu'une partie, en général très minoritaire, les ISBLSM.

Le compte des ISBLSM fait donc partie intégrante du cadre central, alors que les concepts du compte des ISBL n'ont été formalisés qu'en 2002, par le Manuel de l'ONU. L'Union européenne s'apprête à reprendre cette démarche à son compte et à mettre la production de comptes des ISBL au rang d'obligation statistique des Etats-membres.

La Belgique fait partie des pays précurseurs en ce domaine, grâce à l'existence d'une enquête annuelle sur les comptes des associations, mise en place en 1995 pour les besoins du chiffrage du compte des ISBLSM - initiative heureuse qui n'a pas son pendant chez nous.

On peut situer l'objectif de construction d'un compte des ISBL en France comme un moyen terme entre deux demandes déjà anciennes:

- l'une, a minima, pour les besoins du cadre central de la comptabilité nationale, d'améliorer significativement les procédures de construction du compte des ISBLSM;

- l'autre, plus ambitieuse et certainement plus porteuse de sens, de construire un compte satellite pour l'ensemble de l'économie sociale, c'est-à-dire en première approximation les ISBL plus les coopératives et les mutuelles.

\section{ISBL et associations}

Les modalités d'application du statut juridique associatif peuvent être très différentes d'un pays à l'autre; d'ailleurs, en France, trois départements (Alsace ${ }^{(1)}$ et Moselle) font exception à la loi de 1901. Dès lors, si la Belgique a choisi de limiter dans un premier temps son projet aux associations belges (ASBL), il ne faut pas en conclure que le projet français qui ne fait pas explicitement référence aux associations est ou fondamentalement différent ou plus étendu. En France, en première analyse, les ISBL couvrent à la fois les associations ayant une activité économique significative, ou du moins décelable, ainsi que d'autres catégories juridiques (fondations, syndicats, partis politiques...) dont la liste précise reste à établir de façon définitive. Nous n’aborderons pas ici (ce serait prématuré) les questions techniques relatives à la délimitation opératoire des populations que nous entendons décrire dans le compte satellite des ISBL.

\section{La définition de I'ISBL dans le cadre central des comptes nationaux}

Dans toute investigation de nature statistique, il est particulièrement important de commencer par définir avec précision le champ que l'on entend couvrir. Une telle définition doit avoir une base conceptuelle claire et être aisément applicable à la réalité.

Selon le SEC-1995 [3.31], " une institution sans but lucratif(ISBL) est une personne morale (juridique ou sociale) créée pour produire des biens ou des services et à laquelle son statut interdit de procurer un revenu, un profit ou tout autre gain financier à l'unité qui la crée, la contrôle ou la finance ". 
Il faut comprendre l'interdiction de procurer un revenu au sens où il est interdit de procurer un revenu à une unité en sa qualité de créateur, de contrôleur ou de financeur de l'ISBL. Le SEC n'envisage à aucun endroit l'interdiction pour une ISBL de verser des revenus du travail ou des revenus contractuels $d u$ capital (intérêts sur prêts) à des personnes qui, par ailleurs, l'ont créée, la contrôlent ou la dirigent [SYB].

La définition du caractère "non lucratif " d'une activité est et restera un débat sans fin, car on y mêle inévitablement des critères moraux, des critères comptables et des critères économiques. Il revient justement au statisticien d'éclairer cette question récurrente, de l'objectiver par la production d'indicateurs chiffrés inattaquables. Rien noblige en effet une ISBL à être un modèle de vertu ou de dévouement; elle fera partie du champ, dès lors que ses résultats ne sont pas distribués aux apporteurs de capitaux, mais cela n'empêchera en rien ceux-ci de pouvoir se distribuer des salaires sans commune mesure avec les normes du marché. On voit bien sur cet exemple qu'un tableau sur la répartition du résultat gagnera à être complété par un autre décrivant la répartition des salaires et gratifications [PHK].

Le vocabulaire n'est pas neutre. Quand on prend les mots à la lettre, ce qu'ils évoquent ne correspond pas d'emblée à la définition qu'on veut en donner. Ainsi en anglais, les NPOs ou NPIs (nonprofit organizations ou institutions) ne sont pas des entreprises et ne font pas de profits. En français, les ISBL (institutions sans but lucratif) ne sont pas non plus des entreprises, mais peuvent faire des profits; simplement, ce n'est pas leur but. Mais dans le monde Sirene, toutes ces unités sont des entreprises. Dans l'esprit anglo-saxon, dans celui des nouvelles normes comptables IAS, une entreprise ne peut être que lucrative. Cependant, il serait trop simplificateur de faire un lien automatique entre "n'être pas une entreprise " et "n'avoir pas de but lucratif". Aux deux extrêmes, les choses sont claires: une ceuvre sociale purement caritative n'est pas une entreprise, alors qu'une société cotée en Bourse revendique son caractère lucratif. Mais entre les deux on trouvera toutes les nuances intermédiaires. Et quand les coopératives s'affirment sans but lucratif, au motif que leurs réserves sont impartageables, le mot "lucratif " n'a manifestement pas pour elles le même sens que quand il est utilisé par une association de bienfaisance. Il n'en est pas moins légitime [PHK].

Dans l'introduction de son approche institutionnelle, le SCN-1993 [4.17] admet d'emblée que les ISBL different intrinsèquement des ménages, des sociétés et des administrations publiques en signalant que leurs objectifs, fonctions et comportements économiques different également.

\section{Le caractère institutionnel}

Tout d'abord, dans le concept d'ISBL, il faut souligner la référence à la notion d'institution. Cette référence vise notamment à distinguer l'ISBL du concept de ménage, que l'on définit comme "un petit groupe de personnes qui partagent le même logement, qui mettent en commun une partie ou la totalité de leur revenu et de leur patrimoine et qui consomment collectivement certains types de biens et services, principalement de la nourriture et des services 
de logement " [SCN-1993, 4-132]. Si l'acquisition de la personnalité juridique consacre le caractère institutionnel d'une ISBL, le SEC-1995 reconnaît cependant l'existence d'ISBL sans personnalité juridique [SEC-1995 (2.76)].

On notera dès à présent que la formulation relative à la personnalité de l'ISBL n'est pas heureuse dans le contexte législatif belge, où l'on distingue des personnes juridiques physiques ou morales. La personnalité sociale n'est pas une notion utilisée dans notre pays [SYB].

En France, l'association de fait, c'est-à-dire en pratique l'association qui n'est pas déclarée en préfecture dans le cadre de la loi de 1901, est une réalité reconnue par les services fiscaux, peut-être aussi par l'Urssaf (c'est à préciser). Il ne peut s'agir en tout cas que de survivances plus ou moins tacitement tolérées, car aujourd'hui il est impossible de faire fonctionner une association, ne serait-ce que de disposer d'un chéquier, si l'on n’a pas de statuts déposés en préfecture [PHK].

\section{La non-lucrativité}

En second lieu, la définition de la comptabilité nationale met en avant le rôle de producteur des ISBL et démarque ces organisations des sociétés en insistant sur leur absence de lucrativité: "Si les activités de production d'une ISBL génèrent naturellement un excédent ou un défcit, aucune autre unité institutionnelle ne peut s'approprier l'éventuel excédent" [SEC-1995 (3.31)], alors que "la totalité du profit ou du revenu d'une société bénéficie en dernier ressort à d'autres unités institutionnelles, à savoir ses actionnaires. Ceci différencie une société d'une institution sans but lucratif " [SCN-1993, 4.25].

Le SCN-1993 [4.56] précise également que cette absence de lucrativité ne porte pas sur la réalisation d'un excédent d'exploitation positif, mais sur l'affectation de celui-ci.

\section{L'appartenance au secteur privé}

Enfin, le comptable national n'affirme pas d'emblée que l'ISBL est un producteur du secteur privé. Au contraire, il admet l'existence d'ISBL « étatiques » qui, en réalité, remplissent des fonctions pour l'Etat, mais jouissent d'une plus grande indépendance vis-à-vis des pressions politiques [SCN1993 (4.62)].

Ainsi, l'ISBL contrôlée et majoritairement financée par une administration publique sera considérée comme un producteur public; en sa qualité de producteur non marchand public, elle sera assimilée au secteur institutionnel des administrations publiques, le S13 [SEC-1995 (3.28)]. Elle garde toutefois sa qualité d'ISBL et n'en devient pas pour autant une administration publique puisque, par administration publique, le SCN-1993 entend un type particulier "d'entités juridiques, instituées par décisions politiques, qui exercent un pouvoir législatif, judiciaire ou exécutif sur d'autres unités institutionnelles dans un espace donné" [SCN$1993(4.10)]$. 
L'institution sans but lucratif au service des ménages

La comptabilité nationale distingue un type d'ISBL particulièrement différent des autres organisations puisque, à côté des secteurs institutionnels traditionnels, elle a prévu la mise sur pied explicite d'un secteur institutionnel des " institutions sans but lucratif au service des ménages " (ISBLSM). Seules les ISBL qui sont passées au travers de trois filtres y trouvent leur place. Les autres ISBL sont classées dans les secteurs institutionnels des sociétés, des administrations publiques ou des ménages. La procédure du SCN-1993 est similaire à celle du SEC-1995; les termes utilisés sont quelque peu différents, mais le classement qui en résulte est identique. Pour plus de détails, voir SCN-1993 [4.54 à 4.67]; le tableau ci-dessous décrit ce procédé de filtrages successifs.

\begin{tabular}{|c|c|c|c|}
\hline \multicolumn{4}{|c|}{ La définition des ISBLSM par filtrages successifs des ISBL } \\
\hline & \multicolumn{3}{|c|}{ TOUTES LES ISBL } \\
\hline \multicolumn{4}{|c|}{ Filtre 1: Importance } \\
\hline \multicolumn{3}{|c|}{ ISBL avec emploi rémunéré } & $\begin{array}{l}\text { ISBL sans emploi rémunéré } \\
\text { S14 }\end{array}$ \\
\hline \multicolumn{3}{|c|}{ Filtre 2: Nature des ressources } & \\
\hline \multirow{3}{*}{$\begin{array}{l}\text { ISBL marchandes } \\
\quad \text { S11 - S12 }\end{array}$} & \multicolumn{2}{|c|}{ ISBL non marchandes } & \\
\hline & \multicolumn{2}{|c|}{$\begin{array}{l}\text { Filtre 3: Contrôle } \\
\text { et financement publics }\end{array}$} & \\
\hline & $\begin{array}{l}\text { ISBL contrôlées } \\
\text { et financées } \\
\text { majoritairement } \\
\text { par les AP } \\
\text { S13 }\end{array}$ & $\begin{array}{c}\text { Autres ISBL } \\
=\text { ISBLSM } \\
\text { S15 }\end{array}$ & \\
\hline
\end{tabular}

Premièrement, les ISBL qui n'ont pas de personnalité juridique ou qui sont d'importance mineure (parce que, par exemple, elles n'emploient pas de travailleurs rémunérés) sont écartées et assimilées au secteur des ménages (S14). En Belgique, les associations de fait et les ASBL sans emploi rémunéré sont par conséquent classées dans le S14. L'Institut des comptes nationaux fait deux exceptions à cette règle: les syndicats sont classés en S15 et les fonds de pension en S12 [SYB].

Faire de l'absence d'emploi rémunéré le critère décisif et unique d'intégration au secteur des ménages est trop simplificateur. Certes, la présence dans les DADS identifie simplement les associations employeurs, mais il convient de distinguer 
le soubaitable (les principes les plus pertinents) du possible (ce qui est accessible compte tenu des sources disponibles). Or, il existe de nombreux cas où absence de salariés et assimilation aux ménages ne vont pas de pair. D'une part, des associations ou d'autres ISBL ont pu être amenées à créer l'équivalent de " holdings" sans salariés, recevant et distribuant des ressources importantes. L'enregistrement des salariés à un niveau fédéral plutôt qu'à celui d'associations participantes à un réseau ne fait pas pour autant de celles-ci des prolongements d'activités des ménages. Il existe par ailleurs de nombreuses activités gérées uniquement par des bénévoles (sociétés de chasse ou de pêche, groupements de collectionneurs...) qui brassent des sommes non négligeables. L'existence de personnels mis à disposition vient également brouiller les choses; enfin, on ne peut sortir de la sphère des ménages les associations dont les seuls salariés sont des femmes de ménage ou des aides rémunérés par le chèque emploi service. C'est pourquoi je considère que le critère pertinent doit être non pas l'existence de salariés, mais celle d'une activité économique significative qui ne se réduise pas à la simple consolidation de consommations privées des membres pris en tant qu'individus [PHK].

Ensuite, les ISBL dont la production est vendue à des prix économiquement significatifs sont considérées comme des producteurs marchands et, à ce titre, sont affectées aux secteurs des sociétés non financières (S11) ou financières (S12), en fonction de leur activité. Selon le SCN-1993, les prix sont économiquement significatifs quand ils ont une influence significative sur les quantités que les producteurs désirent offrir et sur les quantités que les acheteurs désirent acquérir [SCN-1993 (6.45)].

Cette notion a été traduite par le SEC-1995 en termes opérationnels: un prix est économiquement significatif « à partir du moment où le produit de la vente couvre plus de $50 \%$ des coûts de production " [SEC-1995 (3.19)].

La présence d'ISBL au sein des institutions financières est certainement marginale en termes de production, mais elle se justifie pleinement et il ne faut pas la négliger. Je pense aux réseaux de crédit solidaire, aux "Cigales »... et peut-être aux monts de piété [PHK].

Parmi les ISBL non marchandes (dont la production est fournie gratuitement ou à des prix économiquement non significatifs), on considérera comme faisant partie du secteur des administrations publiques (S13) celles qui sont contrôlées et principalement financées par une administration publique. L'ensemble résiduel est alors rassemblé dans le $S 15$.

Conformément à la description que le SEC-1995 en donne, le secteur des ISBLSM (S15) ne rassemble plus que les « unités dotées de la personnalité juridique (et qui ne sont pas de faible importance) qui servent les ménages et sont d'autres producteurs non marchands privés " [SEC-1995 (2.87 et 2.88)]: c'est la zone cernée par le double encadré du tableau.

Les trois filtres prévus sont bien contenus dans cette définition: importance, nature non marchande des ressources, absence de contrôle et de financement public majoritaire, exprimée ici par l'appartenance au secteur privé (dans le SEC-1995, la distinction entre privé et public se rapporte essentiellement à la question du contrôle). 
Les conventions qui découlent de l'utilisation combinée d'un critère d'importance, d'un critère de ressources et d'un critère d'autonomie sont telles que l'on peut trouver des ISBL dans chacun des cinq secteurs institutionnels résidents distingués par le SEC-1995.

Le SEC-1995 distingue un sixième secteur qui reprend l'ensemble des unités institutionnelles non résidentes. Nous n'envisagerons pas ici le classement institutionnel des ASBL non résidentes [SYB].

Je marque ici une différence d'appréciation avec Sybille. Je ne vois aucune raison de passer sous silence les ISBL de statut étranger qui ont une activité de production sur le sol national. Il y en a de toutes sortes, depuis les "ONG multinationales" jusqu'aux employeurs des jardiniers des cimetières militaires alliés. Je pense également que la prise en compte des organisations confessionnelles, prévue par le manuel de l'ONU, nous obligera à réserver un traitement spécifique pour les structures manifestement étrangères [PHK].

De toute évidence, la comptabilité nationale ne prétend pas au rassemblement des organisations privées non lucratives au sein d'un seul secteur institutionnel. Au contraire, les conventions en vigueur et leur mise en pratique provoquent l'éclatement du secteur des associations entre les différents secteurs institutionnels et, partant, en compromettent la visibilité. D'ailleurs, comme l'a écrit Edith Archambault en 1998, il semble que l'existence du secteur des ISBLSM dans les comptes nationaux ne s'explique que par le souci de débarrasser les comptes des ménages de toute influence parasitaire.

Le SCN reconnait cette faiblesse: "A l'exception des institutions sans but lucratif, toutes les unités institutionnelles d'un type particulier sont rassemblées dans un même secteur" [SCN-1993 (4.13)]. Le secteur des ISBLSM (S15) est le seul qui ne regroupe que des ISBL. Dans les autres secteurs, celles-ci sont amalgamées à d'autres organisations (sociétés, ménages ou administrations publiques) et il est impossible de les isoler. Ce résultat est une des raisons qui justifient la construction d'un compte satellite des ISBL; on s'écartera donc du seul secteur des ISBLSM pour envisager plus globalement l'ensemble des ISBL.

\section{La définition retenue dans le cadre du compte satellite des ISBL}

L'ensemble constitué de toutes les ISBL entendues au sens des conventions de la comptabilité nationale correspond relativement bien au champ que l'on entend couvrir dans un compte satellite des associations en Belgique. Il semble cependant que ces conventions n'établissent pas toujours suffisamment clairement la ligne de démarcation entre les ISBL, les sociétés et les organisations étatiques. Pour répondre à cette faiblesse, le manuel adopté par l'ONU recommande d'utiliser une définition plus précise du secteur sans but lucratif.

Celui-ci se compose :

- d'organisations;

- qui sont non lucratives et, par loi ou par coutume, ne distribuent pas de surplus à ceux qui en sont propriétaires ou les contrôlent; 
- qui sont institutionnellement séparées des administrations publiques;

- qui sont maîtresses de leur gestion;

- auxquelles l'adhésion n'est pas obligatoire.

Reprenons successivement ces cinq caractéristiques et voyons en quoi elles affinent la définition classique des ISBL.

\section{Des organisations}

Selon le Manuel, une ISBL doit exister institutionnellement. Le compte satellite n'entend pas couvrir les rassemblements occasionnels de personnes, sans réelle structure ni identité propre. Parmi les éléments qui prouvent l'existence institutionnelle, on retrouve bien entendu l'acquisition de la personnalité juridique. Celle-ci n'est toutefois pas obligatoire et l'existence d'une structure organisationnelle, la continuité des objectifs et des activités, la délimitation claire de l'organisation, la constitution et le respect d'une charte peuvent constituer des indices sérieux d'existence institutionnelle. Cette première caractéristique traduit donc en termes plus concrets un élément déjà présent dans la définition du cadre central.

\section{Une finalité non lucrative et une contrainte de non-distribution du profit}

Le Manuel réaffirme explicitement la non-lucrativité des ISBL, tant dans leur finalité ("not-for-profit ») que dans leur politique d'affectation des excédents ("non-profit distributing "). Cette double interprétation vise tout d'abord à exclure les fausses institutions sans but lucratif soumises par leurs statuts à une non-distribution des profits, mais procédant, dans la pratique, à une distribution implicite sous la forme, par exemple, de salaires excessifs.

D'autre part, l'affirmation d'une finalité non lucrative permet également d'écarter les organisations qui auraient par erreur adopté une forme juridique non lucrative. D'un point de vue opérationnel, cela ne signifie pas pour autant qu'il soit aisé d'identifier et d'exclure les "fausses " ISBL. Le Manuel souligne également que la non-lucrativité ne signifie pas l'absence de profit ni l'absence de recours à des ressources marchandes.

On ne comprend pas très bien pourquoi le Manuel évoque les propriétaires d'une ISBL qui, par essence, est une organisation sans propriété: personne n’y possède de droits sur les revenus résiduels dégagés.

\section{La séparation institutionnelle}

\section{par rapport aux administrations publiques}

Au sens du Manuel, l'ISBL est une organisation institutionnellement séparée de l'appareil d'Etat et ne jouit donc pas de l'autorité réservée à la puissance publique. A nouveau, si cet élément n'était pas précisé par le SEC-1995 dans sa définition de l'ISBL, on pouvait le déduire de la définition donnée au terme " administration publique " (voir plus haut). Le Manuel en précise toutefois la portée opérationnelle: "L'ISBL peut recevoir un soutien financier important de la part d'administrations publiques et peut compter des représentants de ces administrations dans l'organe qui la contrôle. "C'est donc 
bien un critère de nature fonctionnelle déjà implicitement repris par le SEC-1995 qui est utilisé ici [Manuel, (2.14)].

\section{L'autonomie de gestion}

L'autonomie de gestion ajoute à la dimension fonctionnelle relevée ci-dessus un critère de contrôle. Cela signifie que l'ISBL est capable de contrôler ses propres activités et n'est pas soumise au contrôle d'une autre unité. Par contrôle, le Manuel n'entend ni un critère d'origine (une autre unité peut être à l'origine de la création d'une ISBL), ni le degré de régulation publique auquel seraient soumises les activités de l'ISBL, ni encore la dépendance de l'ISBL vis-à-vis de certaines sources de revenus. Le contrôle peut prendre la forme d'une participation majoritaire (des représentants d'une société ou d'une administration publique) au conseil d'administration ou d'une capacité à nommer la direction de l'organisation.

Ces éléments sont précisément repris par le SEC-1995, qui définit le contrôle exercé par une unité institutionnelle comme étant soit la capacité de détenir ou de contrôler plus de la moitié des voix associées aux décisions, soit simplement le pouvoir de déterminer la politique d'une organisation ou d'en nommer les administrateurs, en s'appuyant sur une loi, un décret ou un statut spécifique [SEC-1995 (2.26)]. Le Manuel s'écarte ici notablement de la comptabilité nationale, puisqu'il n'envisage pas de retenir dans la population des ISBL les organisations qui seraient soumises à un contrôle exercé par une société ou une administration publique. Par conséquent, il écarte notamment de son champ d'application la majorité des ISBL classées dans le secteur institutionnel des administrations publiques. Où commence, où sarrête le contrôle? A ce niveau de dispute, non seulement le statisticien se déclare incompétent, mais même le juriste se doit de le faire. Tout devient affaire de jurisprudence. Lorsqu'on voit (en France) des associations propriétaires en toute légalité de biens immobiliers, pourvues de tous les titres de possession nécessaires, s'en faire dessaisir par les tribunaux au moment où elles allaient les vendre, sous prétexte que, financées avec les surplus d'un budget alimenté par de l'argent public, ces propriétés reviennent à l'Etat, on peut se dire que l'absence de contrôle des années durant ne préjuge en rien du moment où ce contrôle viendra s'exercer, et de la manière la plus discrétionnaire qui soit! Nous ne pouvons donc que nous contenter de critères subjectifs "raisonnables". Car si l'on pousse trop loin la chasse au contrôle, on n'acceptera de fait aucune ISBL dans le secteur des administrations publiques (APU) [PHK].

\section{La liberté d'adhésion}

Enfin, le Manuel stipule que l'adhésion et les éventuelles contributions (en temps ou en espèces) à une ISBL doivent être réalisées sur une base volontaire. Bien sûr, l'organisation peut exiger de ceux qui en bénéficient qu'ils soient membres, tout comme la bonne gestion de l'ISBL peut nécessiter la mobilisation de ressources en provenance des membres, mais tant l'adhésion que l'appel aux contributions ne peuvent être imposés légalement. 
Sous-jacente à la liberté d'adhésion, il y a la question de l'adhésion ellemême. Même si cela n'est pas repris explicitement dans le Manuel, l'ISBL est basée sur la notion de contrat ou de convention entre ses membres. Dès que la notion institutionnelle devient dominante par rapport aux aspects contractuels, on n'est plus en présence d'une ISBL.

La délimitation de la population retenue par le Manuel conduit à exclure certaines organisations considérées par la comptabilité nationale comme des institutions sans but lucratif, à savoir les ISBL contrôlées par les administrations publiques ou par les sociétés et celles auprès desquelles l'adhésion est obligatoire. Le tableau ci-dessous illustre le recentrage de population opéré dans le cadre du projet de compte satellite des ISBL.

La liberté d'adhésion ne peut sentendre qu'en creux: "Nul ne peut être tenu d'adhérer. "En revanche, elle ne saurait sappliquer littéralement, car toute ISBL a le droit si elle le souhaite de mettre des barrières à l'adhésion. Au moins en France, l'esprit de la loi de 1901 est celui de contrat privé, liant deux parties consentantes. Pour devenir membre, il ne suffit pas de vouloir adhérer, il faut être accepté, et l'ISBL a tout pouvoir de définir elle-même ses critères d'entrée [PHK].

\section{La délimitation du secteur des ISBL}

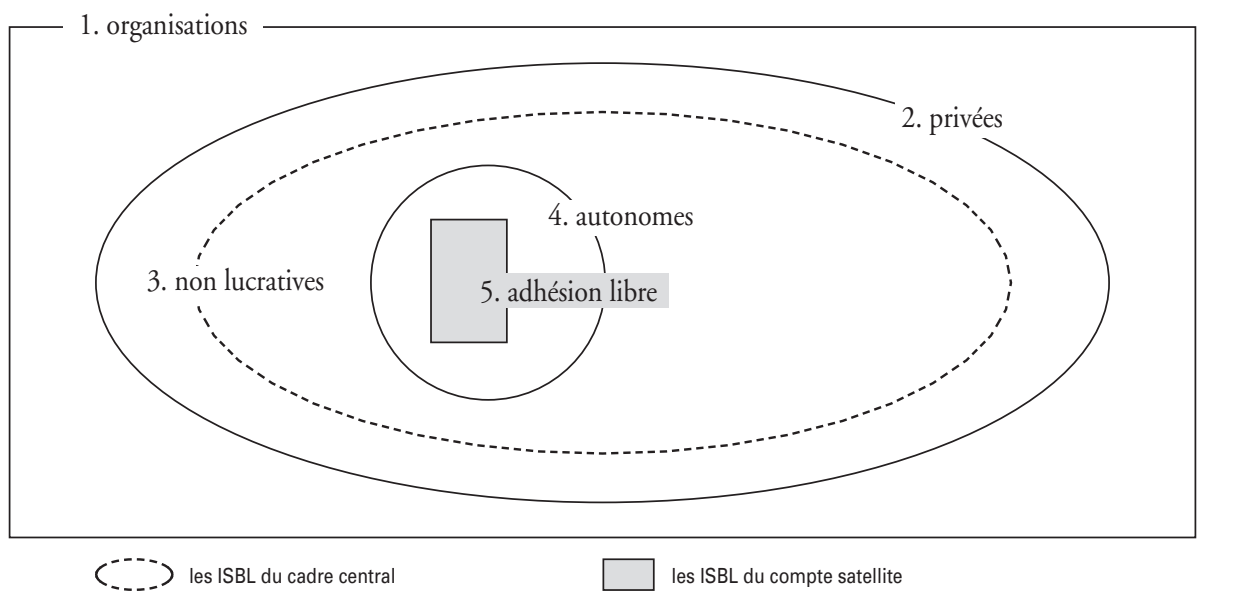

L'ensemble retenu par le Manuel est donc plus large que celui des ISBLSM (qui y sont reprises intégralement), mais plus étroit que celui correspondant à toutes les ISBL au sens de la comptabilité nationale.

Je ne crois pas que nous devions nous sentir engagés au pied de la lettre par les définitions du Manuel, dont le schéma ci-dessus montre bien les implications en termes de délimitation du champ. En effet, bien que fruit d'une collaboration entre un grand nombre de pays, le Manuel cache mal son inspiration anglo-saxonne et nord-américaine. Ainsi exclut-il d'emblée de son analyse les coopératives et mutuelles au titre que " généralement, celles-ci distribuent des 
(2) Inspection générale des affaires sociales. dividendes à leurs actionnaires ", ce qui représente en Europe un contresens absolu.

Au sens du schéma, nos coopératives et nos mutuelles devraient figurer au centre, dans le noyau dur. De la même façon, la transposition directe des conventions du Manuel à la société française, dans des domaines aussi massifs que l'enseignement supérieur, la santé ou la protection sociale, se heurte d'emblée à des difficultés majeures. Il n'y a rien de commun entre la "philanthropie " et l'aide sociale telle que nous la connaissons. En revanche, le Manuel nous indique bien ce que sont les critères d'analyse qu'il faut privilégier, ceux pour lesquels il faut imaginer des indicateurs.

On ne peut pas dire que, sil y a Igas ${ }^{(2)}$, il n'y a pas autonomie de gestion, donc pas d'ISBL; mais il faut donner à l'utilisateur les éléments d'appréciation nécessaires pour situer "nos" ISBL par rapport à celles d'outre-Atlantique [PHK].

\section{Les ISBL et ISBLSM par rapport aux associations et à l'économie sociale}

(3) Les compétences de la Charity Commission s'étendent à l'Angleterre et au pays de Galles. Toutefois, il existe en Ecosse et en Irlande du Nord des organisations sinon équivalentes, du moins comparables. Ce n'est donc pas pour des motifs géographiques que les ISBLSM ne bénéficient d'aucun traitement spécifique de la part des comptables nationaux britanniques.
ISBL et ISBLSM sont des notions de comptabilité nationale, seules ces dernières faisant l'objet d'un compte proprement dit, le S15. Mais dans le langage courant, en France du moins, on parlera des associations, sans plus de précisions. Enfin, certaines personnes sont plus ou moins familières du concept d'économie sociale, dans lequel elles rangent selon les cas tout ou partie des associations.

Essayons d'y voir plus clair sur les contours et la pertinence de ces différents champs.

Les ISBLSM ont une définition précise et ne constituent en principe qu'une catégorie résiduelle, car les conditions requises (financement majoritairement non marchand et assuré par les ménages), si on les prend à la lettre, ne laissent passer que les petites associations de voisinage et quelques rares grandes structures, notamment dans les activités humanitaires.

Il semble que ce statut résiduel autorise en fait une grande liberté d'interprétation. Dans l'actuelle base de la comptabilité nationale française, on compte en ISBLSM des organismes financés à $100 \%$ sur fonds publics, au prétexte que leurs dirigeants n'étant pas fonctionnaires, ils ne sont pas entièrement contrôlés par l'Etat. Nous avons vu plus haut qu'en Belgique l'ensemble des organismes de protection sociale sont, à l'inverse de ce que nous faisons, classés dans les sociétés. Au Royaume-Uni, où pourtant il existe des sources d'information extrêmement détaillées ${ }^{(3)}$, les ISBLSM ne sont pas isolées des ménages.

Les associations n'ont en commun que leur forme juridique. Laissons pour l'instant de côté les RUP (reconnues d'utilité publique) et les trois départements d'Alsace et de Moselle; il reste un champ dont le seul ciment est la loi de 1901, dont on peut faire bien des choses, mais certainement pas une catégorie statistique.

Cependant, même si chacun met derrière le mot " associations " une signification différente, il faut se plier à l'usage, dès lors qu'il est massif; il y a 
bien une demande d'information sur les associations, certes diffuse, confuse, ambiguë, mais réelle.

Les ISBL sont définies par le Manuel de l'ONU et nous n'y reviendrons pas ici.

Quant à l'économie sociale, objet des attentions permanentes, et d'ailleurs seule raison d'être de l'Addes, si elle n'a pas reçu à ce jour de définition statistique précise, il convient de noter que, depuis sa reconnaissance officielle en 1982, elle a résisté aux épreuves et aux mauvais présages, confortant au fil des ans sa légitimité à être considérée comme un secteur spécifique et clairement identifiable.

Cela posé, c'est de la comparaison de nos quatre ensembles que nous pouvons désormais tirer des enseignements utiles. On remarquera d'abord qu'il existe un ordre d'inclusion (et de taille) relativement bien respecté: ISBLSM > Associations > ISBL > Economie sociale.

Ce ne sont pas là des inclusions strictes, mais des champs de volume croissant qui s'emboîtent pour l'essentiel. Il existe des ISBLSM françaises qui n'ont pas le statut juridique associatif, mais elles en sont de proches cousines. Il existe des associations que l'on ne reprendra pas en ISBL, mais ce sont alors des cas limites de perversion de l'esprit de la loi de 1901. Enfin, certaines ISBL pourraient ne pas être retenues dans l'économie sociale, si l'on privilégie pour celle-ci une définition étroitement laïque. Tout cela reste marginal, au moins en première analyse.

Revenons maintenant sur le groupe le plus hétérogène, celui des associations. On observe assez fréquemment que, lorsque ce terme est employé dans une acception englobante, celui ou celle qui l'utilise ne se réfere en réalité qu'à une seule des fonctions sociales généralement dévolues à des organismes à forme associative, s'accaparant en quelque sorte les autres à titre de renfort implicite et consentant. Pour y voir plus clair, et en simplifiant à l'extrême, nous regrouperons ces fonctions sociales en six grandes familles:

1. Les activités collectives créant du lien social entre leurs membres.

2. Les activités fondées sur les valeurs d'engagement, de générosité et de volontariat.

3. Les activités de délégation de service public, notamment dans l'éducation, la santé et l'action sociale.

4. Les activités de gestion d'équipements collectifs.

5. Les activités de production de services gérées dans un esprit acapitalistique.

6. Les activités d'accompagnement du secteur marchand lucratif.

Cette classification n'a aucune prétention méthodologique. Elle recoupe en partie, et c'est inévitable, les distinctions par activité économique et par

(4) Le Manuel définit la nomenclature spécifique ICNPO (International Classification of non profit organizations), qui comprend douze postes regroupés et trente postes détaillés. objet social $^{\left({ }^{(4)}\right.}$, mais ne vise à illustrer qu'une seule dimension de la vie associative: les relations entre les adhérents et les bénéficiaires, c'est-à-dire le " vécu humain " pratiqué et ressenti à l'intérieur de la structure, ce que l'on a dans l'esprit quand on parle de «sa » vie associative. Et l'on voit qu'il y a là une prodigieuse diversité de situations, pire, une impossibilité à établir des traits communs, à définir une cohérence d'ensemble. 
En termes de comptabilité nationale, les deux premières catégories renvoient aux ISBLSM, les deux suivantes au secteur public et les deux dernières aux sociétés non financières, dans chaque cas avec des nuances et toutes les situations intermédiaires ou mixtes que l'on pourra imaginer.

Le $S 15$ nous intéresse donc quand nous voulons mettre l'accent sur la sociologie, la vie collective, le comportement humain. Il se distinguera des ménages proprement dits dès que les structures sont assez organisées pour avoir leur vie propre et des comptes autonomes. Mais il recouvre, on le voit, une profonde opposition entre des organismes tournés vers les intérêts de leurs membres et des organismes d'action humanitaire en direction de bénéficiaires extérieurs.

On nous objectera que la frontière n'est pas aussi tranchée qu'on peut le croire, et c'est tout à fait exact. Il n'en demeure pas moins qu'entre un club de bridge et le Secours catholique il ne peut y avoir d'homogénéité statistique.

Le compte des ISBLSM, certes plus pertinent que celui de l'ensemble des associations, restera donc non seulement résiduel, mais frappé d'une incapacité congénitale à la cohérence.

Le S13 (compte des administrations publiques) nous intéresse quand nous voyons dans les associations un relais privilégié de l'action de l'Etat et des collectivités territoriales, apprécié tant pour ce quil apporte de souplesse dans la gestion que de capacité à innover et à mobiliser (pas dans tous les cas, mais souvent) des compétences et des énergies bénévoles. Nous sommes alors en présence d'associations qui généralement n'ont pas, ou très peu, de membres et qui n’utilisent la loi de 1901 que par commodité.

Enfin, le S11 (compte des sociétés non financières) nous intéresse quand nous pensons à des associations significativement impliquées dans le marché. Mais on ne peut faire l'impasse sur la profonde opposition qui sépare la cinquième famille, constituée de producteurs de services qui conservent un volet d'activités non marchandes, ou du moins qui restent imprégnés de valeurs d'engagement et de dévouement, et la sixième famille, dont les parties prenantes sont généralement, et péjorativement, qualifiées de "fausses associations", même si l'on y observe parfois une véritable vie démocratique, ce qui montre bien que rien n'est simple en ce bas monde. C'est dans la cinquième famille que l'on trouvera une grande part des associations polyvalentes ou mal définies qui nourrissent les codes $913 \mathrm{E}$ et $853 \mathrm{~K}$, ces véritables poisons de la statistique qui obstruent le frêle passage entre le dénombrement des emplois et les comptes nationaux...

Cela nous montre assez que la partie associative du $S 13$ comme celle du S11 ne constituent pas des champs d'étude satisfaisants. En revanche, si l'on y joint, dans chaque famille, en particulier la quatrième et la sixième, les ISBL non associatives qui jouent un rôle semblable, on obtiendra un ensemble certes fort hétéroclite, mais au moins complet dans chacune de ses composantes. A ce titre, le compte des ISBL, fondé sur un principe unificateur clair (pas de but lucratif, quelles qu’en soient les modalités juridiques ou les finalités sociales) trouve une pertinence des mieux venues. 
Mais nous ne sommes arrivés à cette conclusion qu'en partant de considérants internes à la vie des associations. Revenons aux sources du PIB: si l'un des bénéfices, et non des moindres, à attendre d'un compte des ISBL est de proposer une déclinaison du volume de la production en fonction de modes d'évaluation plus ou moins audacieux de l'apport des activités non marchandes et du bénévolat, il n’en demeure pas moins que la fonction essentielle de la comptabilité nationale est de décrire la production de richesses par l'activité économique.

L'activité économique n'est pas concentrée dans les deux dernières familles d'associations, étendues aux autres ISBL, mais concerne également, à un titre certes moins apparent mais néanmoins bien réel, toutes les ISBL, y compris les ISBLSM.

Or sur le plan de l'analyse économique, le critère d'absence de but lucratif tel que le définit le Manuel de l'ONU s'avère très critiquable. Il y a en effet beaucoup plus de différences, de nature comme de fonctionnement, entre une ISBL marchande et une ISBLSM qu'entre une ISBL marchande et une coopérative de la même activité. Pour parvenir à un ensemble cohérent, défini par des propriétés communes pleinement opératoires, il faut ajouter aux ISBL les coopératives et les mutuelles que le Manuel de l'ONU exclut sous des prétextes assez incompréhensibles dans le cadre français ou européen.

Le champ des ISBL institue une barrière artificielle au milieu d'un continuum juridique et de similitudes manifestes de comportement. Et du coup on ne rentre pas, ou pratiquement pas, dans le S12 (compte des sociétés d'intermédiation financière), alors que c'est là que se trouve, naturellement et historiquement, la production de l'économie sociale la plus importante. En substituant à l'absence de but lucratif le principe du fonctionnement sociétarial, l'économie sociale constitue un champ englobant, pertinent, assurant une comparabilité sans ambiguïtés entre nations et systèmes juridiques différents, alors que le champ des ISBL, qui grâce aux efforts des concepteurs du Manuel bénéficie de bonnes garanties de comparabilité internationale, reste frappé de limitations intrinsèques. Je considère donc que le compte satellite des ISBL, s'il marque un progrès notable par rapport à une situation antérieure où nous n'avions rien, doit être replacé dans une perspective dynamique, comme une simple étape vers le compte satellite de l'économie sociale, objectif que l'Addes a toujours défendu, contre vents et marées.

Dès lors, nos quatre champs se classent ainsi par ordre de pertinence croissante:

Associations > ISBLSM > ISBL > Economie sociale.

avec les caractéristiques suivantes:

- associations : champ hétérogène, hétérodoxe, franco-français;

- ISBLSM : champ pertinent mais hétérogène, comparabilité nulle parce que résiduel;

- ISBL: champ pertinent mais conservant des ambiguïtés, bonne comparabilité;

- économie sociale: champ pleinement pertinent et complet; 
sachant que pour l'instant, grâce au Manuel, seul le champ des ISBL est armé pour l'épreuve des comparaisons internationales et que, pour y être également autorisé, le champ de l'économie sociale devra faire l'objet d'un consensus méthodologique explicite nécessitant un investissement intellectuel d'ampleur comparable.

\section{En guise de conclusion générale}

Le compte satellite des ISBL va dans le sens actuellement souhaité d'une meilleure mesure de l'activité immatérielle. Comparable internationalement, il sera un outil précieux pour toutes les réformes de la protection sociale et du système de santé, les politiques d'action culturelle, de l'environnement, de la ville et du développement local, en bref, pour toutes les actions partenariales en Europe et dans les pays de l'OCDE.

La définition communément admise internationalement d'institutions sans but lucratif est certes plus étroite que celle d'entreprises d'économie sociale, mais rien n'interdit aux pays qui le souhaitent (France, Belgique, Espagne, Italie, par exemple) d'ajouter les coopératives et les mutuelles, mieux connues statistiquement que les associations, pour réaliser enfin le compte satellite de l'économie sociale. Un détour international aura donc été nécessaire pour réaliser un projet conçu il y a plus de vingt ans; entre-temps, le monde est devenu un village... 


\section{Bibliographie}

Archambault Edith (1996), Le secteur sans but lucratif, associations et fondations en France, Paris, Economica.

Archambault Edith (1998), "European System of accounts: the French case" Voluntas, vol. 9, $\mathrm{n}^{\circ}$ 4, pp. 375-385, 1998.

Archambault Edith (2002), « Le travail bénévole en France et en Europe ", Revue française des affaires sociales, $56, \mathrm{n}^{\circ}$ 4, décembre 2002 , pp. 13-39.

Defourny Jacques, Deweltere Patrick et Fonteneau Bénédicte (1999), L'économie sociale au Nord et au Sud, Bruxelles, De Boeck Université.

Gadrey Jean, «L'invention de l'utilité sociale des associations en France : à la recherche de conventions, de régulations, de critères et de méthodes d'évaluation, Colloque du Ciriec Canada, Rimouski, 20-22 mai 2003.

Gide Charles (1920), Les institutions de progrès social, Paris, Sirey.

Kaminski Philippe (1983), « Des chiffres pour l'économie sociale. Les sources d'information; mobiliser les données existantes et en créer de nouvelles ", Paris, Ir colloque de l'Addes, 14 mai 1983.
Neyret Guy, Nivlet Jean-Marie et Rault Daniel (1998), Rapport de la mission du CNIS "Associations régies par la loi de 1901 ", remis en novembre 1998.

Milgrom P. et Roberts J. (1997), Economie, organisation et management, Bruxelles, De Boeck Université.

Mertens Sybille (2002), « Vers un compte satellite des institutions sans but lucratif en Belgique ", thèse de doctorat, université de Liège. Salamon Lester, Anheier Helmut et alii (1999), Global civil society. Dimensions of the nonprofit sector, Baltimore, The Johns Hopkins Center for civil society studies; deuxième édition 2001.

Système de comptabilité nationale (SCN 93), Genève, ONU, 1995.

Système européen de comptes (SEC 95), Luxembourg, Eurostat, 1996.

Tice Helen (2002), Handbook on nonprofit institutions in the system of national accounts (Manuel des institutions sans but lucratif dans le système des comptes nationaux), New York, United Nations Statistical Division.

Vienney Claude et Weber Jean-Charles (1983), «Esquisse pour un compte satellite de l'économie sociale ", Paris, Ir colloque de l'Addes, 14 mai 1983. 